Scientific Visualization, 2021, volume 13, number 1, pages 69 - 82, DOI: 10.26583/sv.13.1.06

\title{
On the development of aeroballistic experiment techniques for flow visualization
}

\author{
S.I. Gerasimov"1,A,B,C,D, V.I. Erofeev,A,D, V.A. Kikeev3,A,D, K.V. Totyshev4,C, E. G. Kosyak5,B, P. \\ G. Kuznetsov'6,B, R.V. Gerasimova7,B
}

A Institute for Problems of Mechanical Engineering, Institute of Applied Physics, Russian Academy of Sciences, Nizhny Novgorod, 603024 Russia

в Sarov State Physics and Technical Institute National Research Nuclear University

MEPhI, Sarov, 607186 Russia

c Russian Federal Nuclear Center All-Russian Research Institute of Experimental Physics, Sarov, 607188 Russia

D Nizhny Novgorod State Technical University, Nizhny Novgorod, 603950 Russia

1 ORCID: 0000-0002-6850-0816, s.i.gerasimov@mail.ru

2 ORCID: 0000-0002-6637-5564, erfo4@mts-nn.ru

3 ORCID: 0000-0002-2375-0803, vkikeev@mail.ru

4 ORCID: oooo-0002-7141-0408, ktotyshev@yandex.ru

5 ORCID: 0000-0001-6291-2396, keg@sarfti.ru

6 ORCID: 0000-0003-2691-206X, kpg@sarfti.ru

7 ORCID: 0000-0002-2711-3975, grv@sarfti.ru

\begin{abstract}
$\underline{\text { Abstract }}$
The schemes of shadow rendering, supplementing the methodology of aeroballistic experiment, consisting in the ejection model, mounted in a special separable pallet, ballistic installation; the separation and capture of the pallet by the clipper; the span of the model with given initial conditions of motion speed and angle of attack on the measuring site aeroballistic tracks; contactless external trajectory registration model of the synchronous photographing models of digital cameras stereophotos, operating in standby mode on the background of reference marks of the coordinate reference system. Photographing takes place at the moment when pulsed light sources are triggered synchronously with the model's movement. Under these conditions, shadow visualization schemes are functional, which represent an upgraded method of a luminous point using a protective lens, a combined method that includes registration in passing light using a camera with an electron-optical shutter, and a shadow background method. Typical registration patterns are given.
\end{abstract}

Keywords: ballistic experiment, aerodynamic characteristics, light-gas installation, photoregistration.

\section{Introduction}

One of the tasks of modern ballistic experiments is to accurately measure the aerodynamic characteristics of bodies moving at super-or hypersonic speeds in the air. The aerodynamic characteristics of free-flying bodies are determined by indirect methods based on the use of a differential relationship between the coefficients of aerodynamic forces and moments and the parameters of the registered motion of the body under study, established by a system of General equations of motion [1-4]. Consideration of the equations shows that the problem of determining the aerodynamic characteristics from the data of trajectory measurements performed in a ballistic experiment is mainly reduced to calculating the first and second derivatives of coordinates included in these equations in time or coordinate. Since these errors are proportional to the coordinate measurement errors, the magnitude of the 
errors in determining the aerodynamic characteristics in a ballistic experiment largely depends on the magnitude of the errors in measuring the coordinates of the model [1].

The traditional formulation of an aeroballistic experiment (AE) on ballistic routes is that a model of an aircraft (AC) is fired from a powder or light-gas ballistic installation (BI) with the initial conditions set by the test program for the number of $M$ and the angle of attack $\alpha$ [5-7]. Synchronously with the flight of the model, using optical means of the range for measuring the kinematic parameters (KP, multiple registration is performed at fixed time points $\mathrm{t}_{\mathrm{i}}$ of the linear (coordinates $\left.\mathrm{X}_{\mathrm{i}}, \mathrm{Y}_{\mathrm{i}}, \mathrm{Z}_{\mathrm{i}}\right)$ and angular $\left(\vartheta_{i}, \psi_{i}, \gamma_{i}\right)$ position of the test object (TO) in space. The sequence of synchronizing commands for triggering pulsed light sources (PLS) that provide photo-registration of the model in flight was developed using an automatic control system for the optical measuring complex equipment [8].

The method of calculating the external vector parameters of the spatial position of the TO consists in equalizing calculations using the least squares method (LSM) of the dependencies between the measured values - coordinates of images and unknown (determined) external vector parameters of the TO [9]. The equalization principle includes a calculation process that is common both for refining the orientation elements of images and for calculating the determined parameters of the TO spatial position . The problem of calculating the parameters of the TO spatial position is solved by drawing up the corresponding equations, in which the known values are substituted:

- updated values for internal and external orientation elements of images;

- approximate values of the parameters of the OI spatial position $\left(X^{0}, Y^{0}, Z^{0}, \vartheta^{0}, \varphi^{0}\right.$, $\left.\gamma^{0}\right)$.Since the equations are non-linear and usually more points are measured than required, the solution is performed using LSM, by minimizing the functional:

where -

$$
\Phi\left(\overline{a_{u}}\right)=\sum_{j=1}^{K}\left(W_{j} \cdot \sum_{i=1}^{N} \overline{\Delta r_{i}^{2}}\right)=\min
$$

$\bar{a}$ - vector of matching parameters-linear and angular coordinates of $\mathrm{TO}(X, Y, Z, \vartheta, \varphi, \gamma)$; $\overline{\Delta r_{i}}$ - the vector of residuals between the measured values of the coordinates of the TO points in the images and those calculated using known formulas;

$\mathrm{Wj}=\left(\begin{array}{cc}\frac{1}{\sigma^{2} x} & 0 \\ 0 & \frac{1}{\sigma^{2} y}\end{array}\right)$ - weight matrix (2x2) of measurements of points' coordinates $x_{i}, y_{i}$ on images (taken independently, with the same weight);

$\mathrm{u}=1,2, \ldots, 6$-index of matching parameters;

$\mathrm{K}$ - the number of digital cameras that measure the coordinates of images of TO marking points $(2 \leq \mathrm{K} \leq 4)$;

$\mathrm{N}$ - the number of TO points measured in the stereo pair images;

$\mathrm{i}, \mathrm{j}$ - indices of summation.

In this case, the position of the TO in space is found, i.e. the $X, Y, Z$ coordinates of the center of gravity and orientation angles $\vartheta, \varphi, \gamma$, at which the coordinates of the images of the reference points of the TO marking on the stereo pair images (left and right and/or upper and lower) coincide with the measured ones.

At each step of the iteration process, the values of the parameters of the previous approximation are calculated and corrected. The calculation is based on the formula:

where:

$$
a_{u}^{v+1}=a_{u}^{v}+\left(G_{u u}^{-1}\right)^{V} \cdot D^{T},
$$

$G^{-1}$ is an inverted matrix (6x6) of coefficients of the system of normal equations (partial derivatives of the calculated values of the coordinates of the image of the TO points in the images based on approximate values of the spatial linear and angular coordinates of the TO); $D$-matrix (1x6) of free terms of the system of normal equations; $v$ - the number of the iteration; 
$\mathrm{T}$ is the transpose index.

The excess of information makes it possible to obtain a statistical estimate of the rootmean-square errors in determining the KP of the TO movement based on the materials of stereophotogrammetric measurements:

$$
\sigma_{a_{u}}=\sqrt{\frac{\Phi_{K}}{4 N-u} G_{u u}^{-1}},
$$

The discrete values of the coordinates of the center of gravity and the angular orientation of the OI in space calculated for the known moments of photographing in the experiment are accepted as the main initial data for the subsequent stage of calculating the aerodynamic characteristics of the TO.

When performing KP measurements, the certified digital cameras Canon are used, the physical resolution of which is known from the passport and is characterized by an image pixel size of 6-8 microns. For photogrammetric image processing, a program is used that provides an "instrumental" error in measuring the coordinates of digital images with subpixel accuracy of 2-3 microns. In this regard, the error of the photogrammetric processing of images are considered to be known values. According to the statistical analysis of materials, the error of coordinate measurements from digital photographs is characterized by a random mean square deviation $\sigma_{\mathrm{x}, \mathrm{y}}=5$-10 microns with a probability $\mathrm{P}=0.95$.

\section{Description of the photo post.}

Photo-recording devices with electron-optical converters (EOC) are widely used for recording fast-moving processes [7]. On the basis of cameras with an EOC, a combined photo post (CPP) was created, which allows to obtain an image of an aerodynamic model in reflected light and a shadow picture of its flow [10]. The presence of shutter systems allows photo registration at any time of the day and with almost any background illumination. CPP using analog cameras is advisable at the initial stage of the shot in more severe conditions of exposure to muzzle exhaust [11]. Digital camera-based CPP is used on the measuring section of the aeroballistic range outside the zone of combustion products spread, after cutting off the pallet elements, etc. Figure 1 shows a diagram of the implementation of this scheme for the intermediate ballistics stage. When conducting an aeroballistic experiment, namely throwing the test model at a speed of $0.5 \cdots 3.0 \mathrm{~km} / \mathrm{s}$ from a ballistic installation (BI), one of the most important tasks of intermediate ballistics is to study the interaction of elements of a complex system: the actual model, the leading elements (pallets, plates, obturators), the barrel of the BI, the combustion products of the powder charge, shock waves generated by moving bodies.

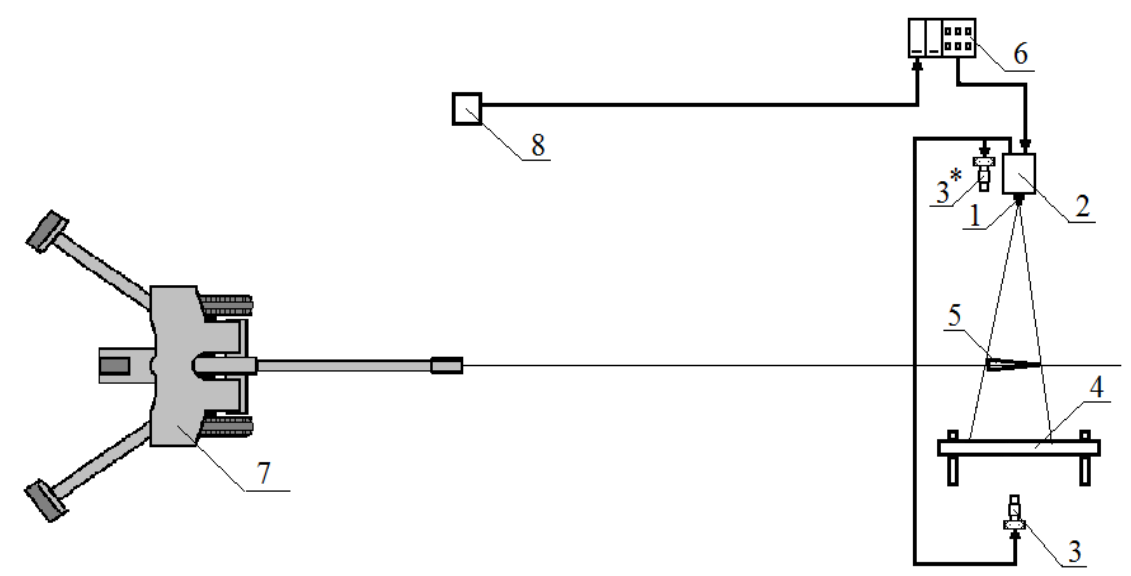

Figure 1 - Two-channel optical registration system: 1 - point emitter; 2 - electric energy storage device; 3 - cameras with an electron-optical shutter; 4 - screen; 5 - model; 6 - synchronization unit; 7 - gun; 8 - photodetector. 
When the BI (7) is fired, a glowing area appears in the muzzle section, which is oriented to the photodetector (8), which starts the circuit with the necessary delay. A gas-discharge point pulse emitter (1) with limited discharge propagation is designed in such a way that, on the one hand, it has the necessary lighting parameters sufficient to build an image of the model in reflected light (brightness $\approx 6.2 \mathrm{Msb}$, luminous body $0.785 \mathrm{sm}^{2}$; with the sensitivity of the film So.8=1000 units, and on the other hand, it has a small enough glow body to be a point source of the shadow scheme and build shadow spectra of satisfactory quality on a matte screen. The emitter (1) is activated by an energy storage device (2) ( $E=150$ joules), generating a light pulse, and simultaneously a synchro pulse is issued to open the shutters of homemade analog cameras (main and additional) EPOS ( 3 and $3^{*}$ ), one of which photographs the shadow image, and the other - in reflected light. Exposure of photographic material (shutter time) - 0.5 ...1 $\mu \mathrm{s}$ [10].

A digital stereo post consists of two digital cameras located on a vertical basis, and two cameras located on a horizontal basis-symmetrically relative to the first two. Digital cameras are located in a closed (lockable) box-safe, which excludes any mechanical and physical effects from possible fragments during experiments and the human factor. On the front wall of the box, there is a hole for the lens with a diameter of 50-60 mm, equipped with an automatic shutter that opens the lens before taking photos and locks it after taking photos. Figure 2 shows characteristic images in reflected light obtained simultaneously by the post cameras with an open shutter during sequential operation of pulsed gas-discharge light sources with duration of 1 microsecond [7].

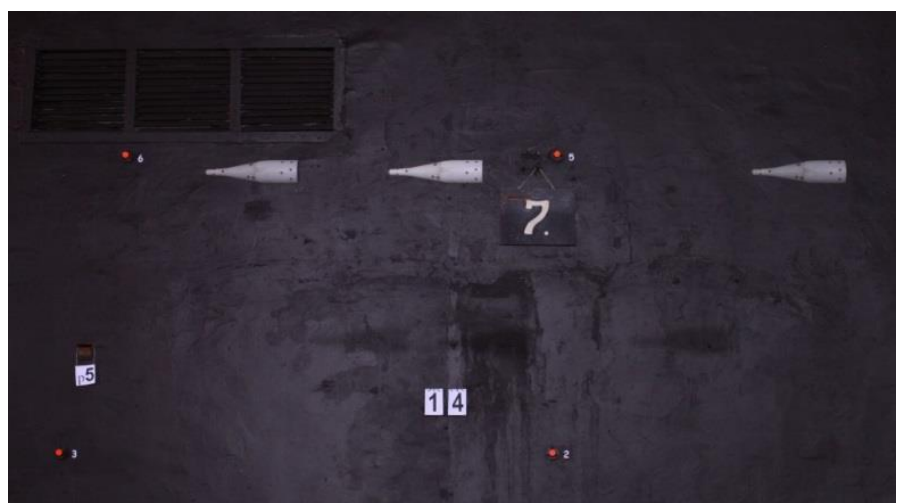

Figure 2 - Example of a camera registering several positions of a model moving at a speed of $1.2 \mathrm{~km} / \mathrm{s}$ in one of the stereo posts.

Figure 3 shows an example of using the scheme in a ballistic experiment in the intermediate ballistics section.
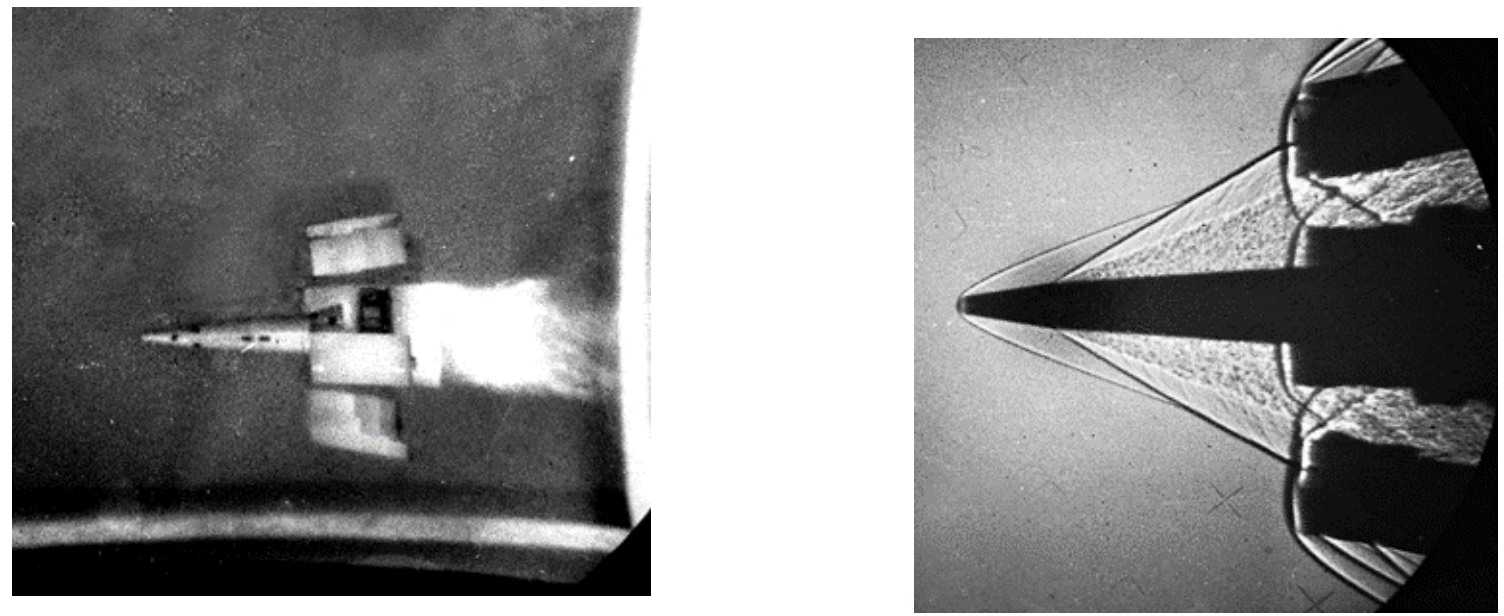

Figure 3 - Movement of assembly elements recorded by analog cameras with EOC. 
All elements of this system are mobile and allow you to organize a combined photo post in almost any conditions of the experiment. At the stage of external ballistics (including on the measuring section of the aeroballistic range), when the pallet elements are cut off, more expensive digital cameras with EOC can be used in this scheme [12 ]. An example of obtaining information when installing a photo post on the measuring section of an aeroballistic shooting range using a software-controlled multi-frame electron-optical complex for high-speed registration of a series of images of a fast-flowing process is shown in Figure 4.

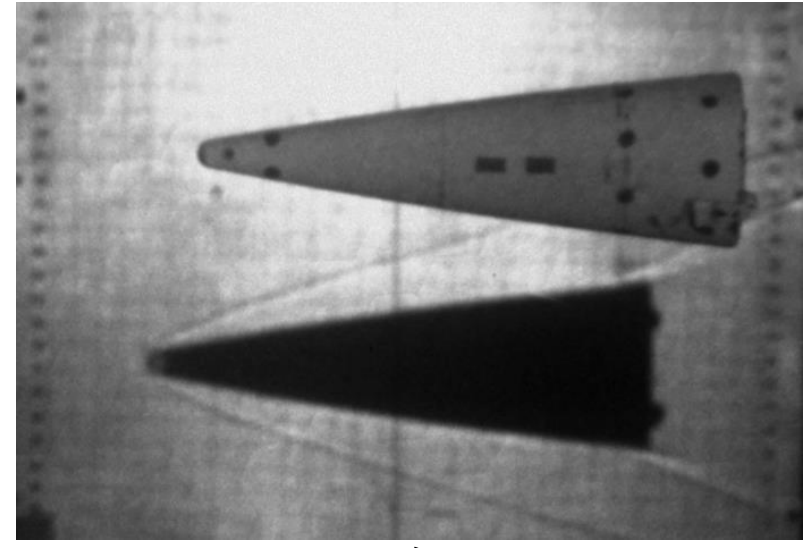

a)

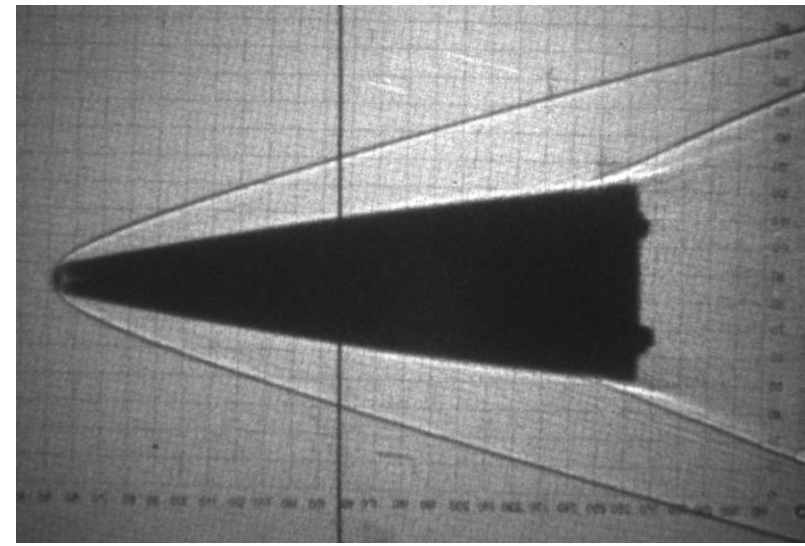

b)

Figure 4 - results of CPP registration: an image of an aerodynamic model moving at a speed of $1480 \mathrm{~m} / \mathrm{s}$ in reflected light (a) and a shadow picture of its flow (b).

The quality of the information obtained allows you to measure external parameters with the accuracy stated above. This, in turn, allows the required accuracy to determine the characteristics of the aerodynamic forces and moments acting on aircraft in flight, for example, $C d(\alpha, M)$ - the ratio of the center of pressure; $C \tau$ - coefficient of tangential force; $C n^{\alpha}-$ the derivative of the coefficient of normal aerodynamic force angle of attack; $m_{z}^{\varpi_{z}}$ the derivative of the coefficient of lateral aerodynamic moment on the dimensionless angular velocity $\varpi_{z}=\omega_{z} \cdot \frac{L}{V}$ (V - speed of flight models).

However, for a number of aircraft, in addition to solving the main problem in AEdetermining the characteristics of aerodynamic forces and moments, there is a need to visualize the flow spectra of the model.

For example, the main aerodynamic feature of the model when using flap controls is the presence of a large normal force and a certain angle of attack at zero transverse moment. This leads to significant radial deviations relative to the main line of aim of the aeroballistic range and the presence of significant balancing angles of attack. In this case, fluctuations in the angle of attack are damped. Rotation of models relative to the longitudinal axis (by $\gamma$ ) due to twisting in the BI barrel (the appearance of the initial angular velocity of rotation of the model relative to the longitudinal axis when moving in the barrel channel) leads to a significant change in the roll angle $\gamma$. Therefore, an essential circumstance in the formulation of aerodynamic experiments with such models is to identify local features of the flow around the aerodynamic controls. Shadow images can provide useful information for studying the flow pattern in this zone, depending on the Mach number and angles $\alpha_{\Pi}$ and $\varphi_{\Pi}$. And since these parameters change on the trajectory and significantly depend on the initial driving conditions and parameters of the test object, you need multi-angle shadow shooting and shooting at different points of the trajectory. The implementation of such features can significantly improve the effectiveness of each individual experiment.

The aerodynamics of an object that is divided into sections are significantly complicated in comparison with non-dividing objects. In this case, in the initial phase of motion there may be a significant aerodynamic relationship between the two sections, but when diverging axially by some distance, the relationship disappears and the features of the second section 
motion in the downward trace of the first section appear. The aerodynamic forces acting on the section are manifested through linear displacements under the action of these aerodynamic forces (the second integral of the force in time), and the aerodynamic moments through angular displacements under the action of these moments (the second integral of the moment in time). These features can significantly complicate the processing of an aeroballistic experiment with models divided into separate flying sections. This is due to the fact that the aerodynamic forces and moments acting on the sections can significantly and non-linearly depend on the linear and angular coordinates that determine their location relative to each other and the velocity vectors. These dependencies may have a qualitatively different character depending on whether the second section is located in the near (to the "neck") or in the far part of the satellite track. These difficulties in the analysis of an aeroballistic experiment with separating objects mean that it is advisable to perform multiple shadow registration of the shadow spectra of the section flow at different points of the trajectory in order to effectively conduct it. This allows us to identify the flow features in different phases of the separation process and to justify the real nature of the aerodynamic dependencies.

The complexity of the problem lies in the fact that the above algorithm for obtaining KP involves obtaining up to hundreds (from dozens of stereo posts) of high-quality images of the tested model in reflected light (for visualization of at least three marks applied to the surface of the model at specified points). The corresponding "background" illumination significantly complicates high-quality shadow shooting and makes it impossible to use classic direct-shadow shooting on open film. Combining the determination of these KP and obtaining a high-quality picture of the shadow spectra of the flow in one experiment is carried out using special registration schemes in transmitted light.

\section{Application of background oriented schlieren method}

The trend of the last decade in the development of scientific research of aerohydrodynamic flows is the rapid convergence of visualization of numerical simulation results with visualization of experimental results. Comparison of digital numerical and experimental flow fields can serve as an effective tool for analyzing aerohydrodynamic processes based on the results of experimental and numerical studies of complex flows. One of such directions in the field of registration of fast-moving processes is the background oriented schlieren (BOS) method [13]. In [14], BOS method was used to visualize regions with a density gradient when the model flows around in a wind tunnel, but there are no studies on the use of BOS method in a direct aerodynamic experiment. Using CPP elements and BOS algorithms, it is possible to visualize areas with a density gradient caused by the movement of an aerodynamic model at supersonic speed. Figure 5 shows the results of registering a moving aerodynamic model using BOS method. Registration was carried out by a camera with an EOC on the background of a contrasting screen with its illumination by a pulsed light source. Additionally, prior to the experiment, a reference (without a model) image of the contrast screen was registered. Processing of two received frames using a crosscorrelation algorithm allowed visualization of the perturbation regions. Processing was performed using the PIVview program (demo version). A multi-pass cross-correlation processing algorithm with a square survey window and 50\% overlap was used. The size of the survey window was iteratively reduced from 256 to 16 pixels. Approximation of the correlation function was performed using three-point Gaussian interpolation without pretreatment images. The fast Fourier transform algorithm was used to calculate the correlation function. The results obtained are presented as the absolute value (b) and the vertical component (c) of the displacement field of image elements caused by the refraction of probing radiation when passing through the perturbation region. 


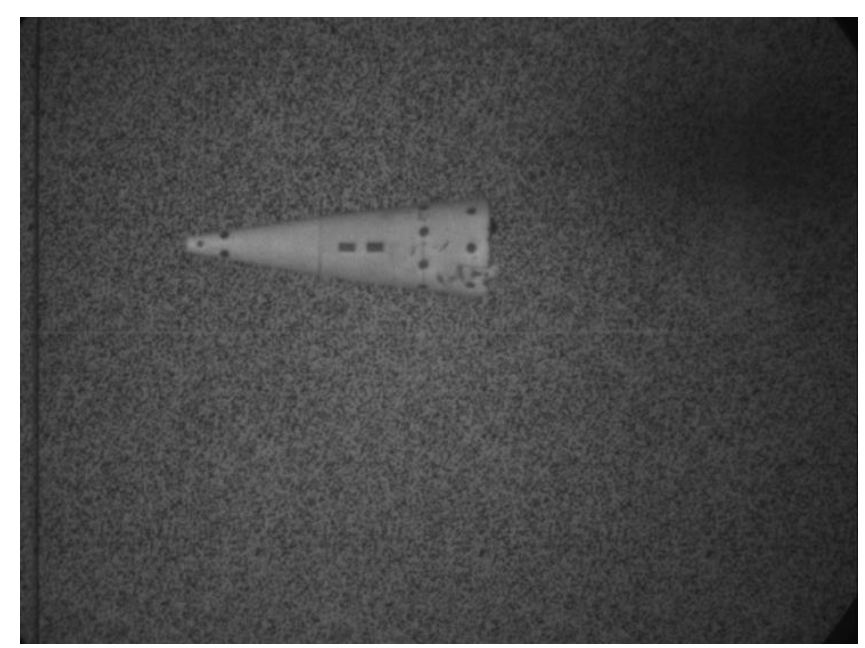

a)

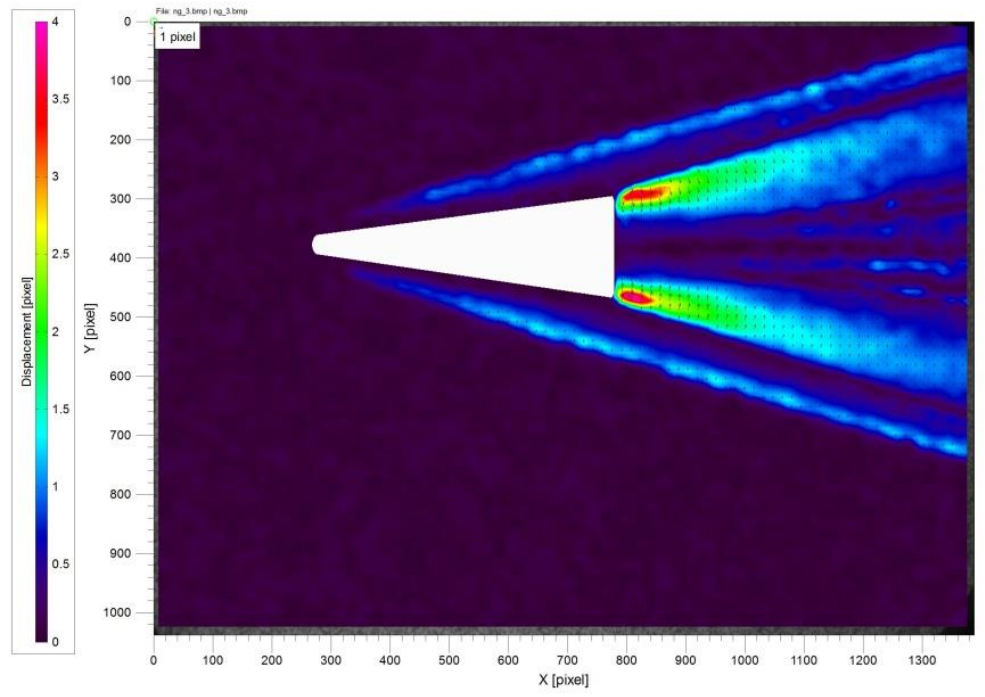

b)

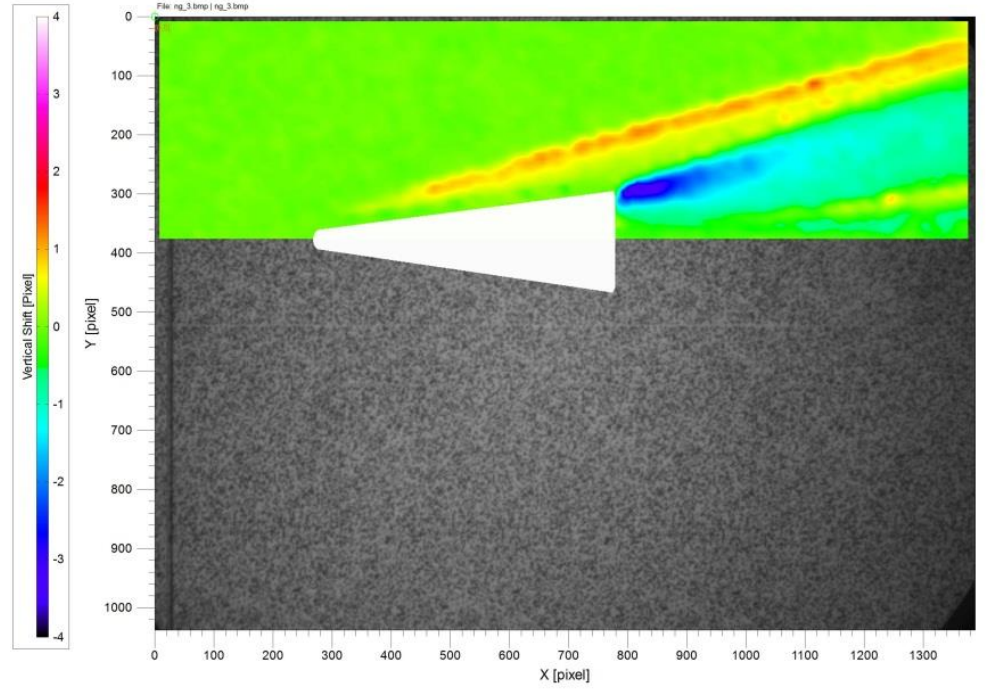

c)

Figure 5 - The result of BOS registration: the original image (a) and the results of crosscorrelation processing $(b, c)$.

Another task that can be solved using BOS method in the experiment is to determine the speed of an OI moving at supersonic speed

When the TO moves at supersonic speed, a curved SW is generally formed in front of it, which, far from the TO, degenerates into a weak (sound) perturbation wave. The method 
[1] is based on the measurement of SW away from the TO. The speed of the TO is calculated using the formula:

where $V_{T O}$ is the speed of the TO;

$$
V_{T O}=\frac{a}{\sin (\alpha)}
$$

$a$ - speed of sound in the air;

$\alpha$-half of the angle of the solution of the head SW.

The shape of the SW in the immediate vicinity of the TO depends on the shape of the body. The method [12] is based on measuring the opening angle of the SW in the immediate vicinity of the conical shape TO. The velocity of the TO is calculated using the formula:

$$
V_{T O}=\frac{a}{\sqrt{\sin (a)^{2}-1,2 \sin (\beta)^{2}}},
$$

where $\beta$ is half the angle of the TO solution of a conical shape.

Thus, in the known methods $[15,16]$, one position of the TO located in the registration zone of the photo recorder is registered and one value of the TO speed is determined. In addition, a typical schema photographic head SW [17] suggest, the presence of elements of the scheme of registration commensurate with the size TO; the use of a point light source; no external (background) illumination or the use of photographic electronic shutter. These factors narrow the scope of the known methods. To eliminate these shortcomings, methods have been developed $[15,16]$. they are based on video recording of an OI moving at supersonic speed against a background that is a surface with a structure of many small details with high optical contrast (artificial background screen, forest, grass, sand, brick wall...). Due to the refraction of the probing beam, the elements of the background screen in its image are shifted when passing the area of the head SW. By analyzing the offsets of elements in the background screen image, the position and shape of the head unit are determined. Based on the measurement of the SW opening angle away from the TO, its speed of movement is determined [4]. In the case of close-up video recording of the movement of a conical TO, its speed is determined based on information about the slope of the oblique shock wave relative to the surface of the TO speed $[18,19]$.

The reliability of the obtained data on the speed of the TO based on high-speed video recording of the process of its movement, subsequent visualization of the SW and analysis of the information obtained was verified experimentally. Video recording of the flight of a $7.62 \times 54$ R PS bullet fired from a Dragunov sniper rifle (DSR) was carried out. Registration was carried out in the free flight area of the bullet, at a distance of $\sim 10 \mathrm{~m}$ from the cross section of the DSR barrel, using a high-speed video camera (shooting frequency $2128 \mathrm{fps}$; exposure time $5 \mu \mathrm{s}$;) against the contrast of the screen width $1.4 \mathrm{~m}$. Figure 6 shows the results of registration of the head SW in supersonic flight during one of the experiments. Using the obtained images of the head SW, by measuring the angle of the SW solution at a distance from the bullet, the values of the bullet velocity $\left(V_{T O}\right)$ are calculated using the formula (1). The results are shown in table 1, which also provides information about the bullet speed obtained using light-blocking cross sections $\left(\mathrm{V}_{B Z U}\right)$. Video recording was carried out at a distance of $\sim 7 \mathrm{~m}$ from the installation site of light-blocking sections. Note that at a distance of $7 \mathrm{~m}$, the drop in bullet speed is $\sim 5 \mathrm{~m} / \mathrm{s}$. The value of the bullet's flight speed, adjusted for this circumstance, is also shown in table 1.

Figure 7 shows one of the frames for recording a conical TO (angle of the solution $\beta=$ $15.3^{\circ}$ ) and the head of the SW. Based on the measurement of the angle of the head SW solution near the TO $\left(\alpha=31.2^{\circ}\right)$, the TO velocity is calculated. The calculated speed value was $1508 \mathrm{~m} / \mathrm{s}$, which is consistent with the speed value obtained using light-blocking sections $(\mathrm{V}=1482 \mathrm{~m} / \mathrm{s})$ within $2 \%$. 


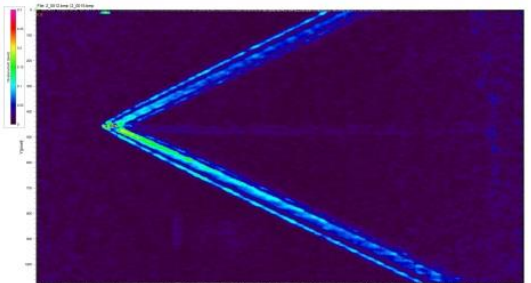

Position 3

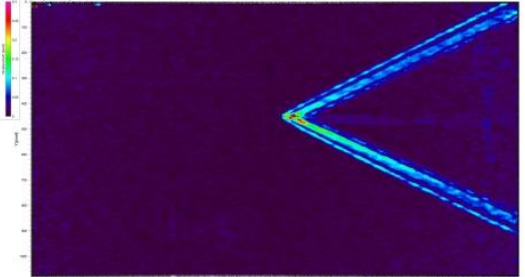

Position 2

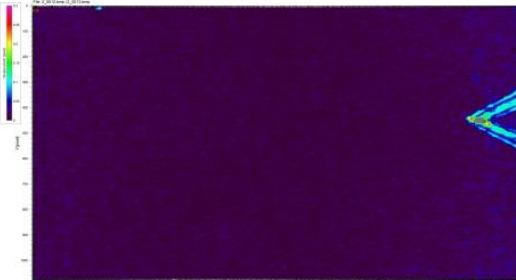

Position 1

Figure 6 - The result of detecting SW from bullet movement at supersonic speed (Exp. № 1).

Table 1 - Measurement results.

\begin{tabular}{|c|c|c|c|c|c|c|}
\hline & & $\alpha^{\circ}$ & $\begin{array}{c}\mathrm{V}_{T O} \\
(\mathrm{~m} / \mathrm{s}) \\
\end{array}$ & $\begin{array}{l}\mathrm{V}_{B Z U} \\
(\mathrm{~m} / \mathrm{s})\end{array}$ & $\begin{array}{c}\mathrm{V}_{\text {cor }} \\
(\mathrm{m} / \mathrm{s})\end{array}$ & $\delta=\frac{V_{c o r}-V_{T O}}{V_{c o r}} \cdot 100 \%$ \\
\hline \multirow{3}{*}{ Exp.№ 1} & Position 1 & 27,1 & 752 & \multirow{3}{*}{807,2} & \multirow{3}{*}{802,2} & 6 \\
\hline & Position 2 & 26,2 & 775,9 & & & 3 \\
\hline & Position 3 & 26,2 & 775,9 & & & 3 \\
\hline \multirow{3}{*}{ Exp.№ 2} & Position 1 & 26,1 & 778,7 & \multirow{3}{*}{808,6} & \multirow{3}{*}{803,6} & 3 \\
\hline & Position 2 & 26,3 & 773,1 & & & 4 \\
\hline & Position 3 & 26,2 & 775,9 & & & 3 \\
\hline \multirow{3}{*}{ Exp.№ 3} & Position 1 & 26,1 & 778,7 & \multirow{3}{*}{813} & \multirow{3}{*}{808} & 4 \\
\hline & Position 2 & 26,1 & 778,7 & & & 4 \\
\hline & Position 3 & 26 & 781,4 & & & 3 \\
\hline
\end{tabular}

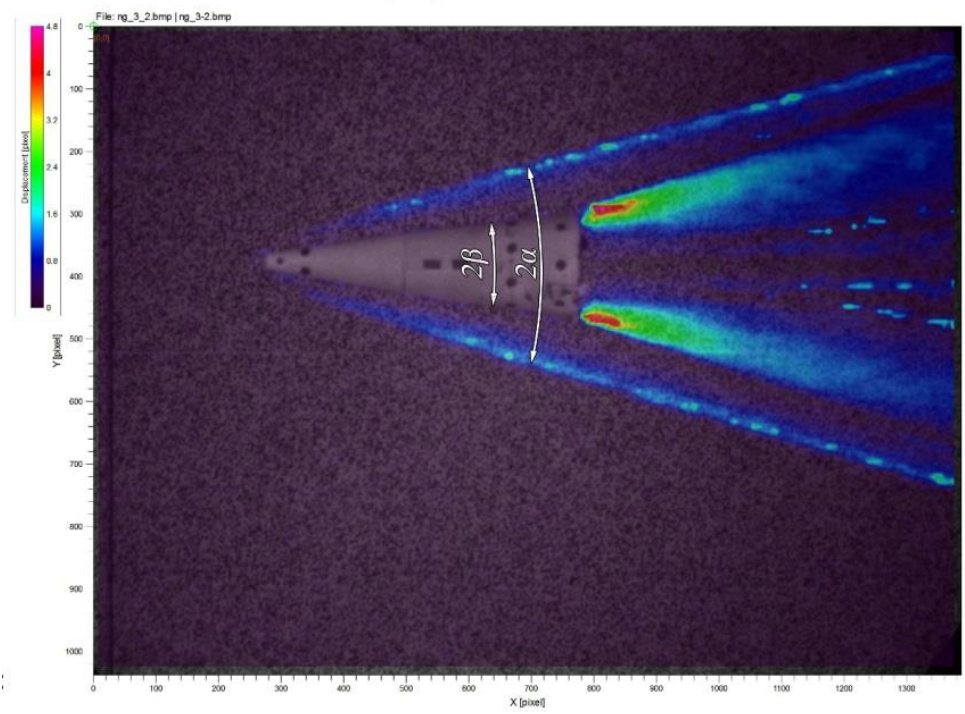

Figure 7 - The result of the registration of SW arising from the movement of the TO at supersonic speed $\left(2 \alpha=31,2^{\circ}, 2 \beta=15,3^{\circ}\right)$.

\section{The method of the luminous point using a protective shell}

The shadow photography stand, which does not use optical elements and is used in an aeroballistic experiment, is used to obtain high-quality shadow spectra of the flow around models with a length and caliber of 8-500 $\mathrm{mm}$ and $8-140 \mathrm{~mm}$, respectively, moving at a speed of $350-3000 \mathrm{~m} / \mathrm{s}$. The stand is mobile and can be installed at any point of the trajectory of models inside the aeroballistic route with the ability to adjust the position of the registration zone in the $\mathrm{Y}$ and $\mathrm{Z}$ coordinates in the range of $\pm 1 \mathrm{~m}$ relative to the axis of the route. The launch of the light sources is from the non-contact sensors; the moment of exposure is recorded by a single chronographic system of the aeroballistic route. The size of 
the registration area is $800 \times 600 \times 600 \mathrm{~mm}$. The resulting shadow image shows the configuration of the head shock wave and weak shock waves( Mach waves), the zone of formation of the boundary layer and its structure, the structure of the bottom trace, and other subtle effects that occur when the flying object flows around. A typical example of a shadow image is shown in Figure 8.

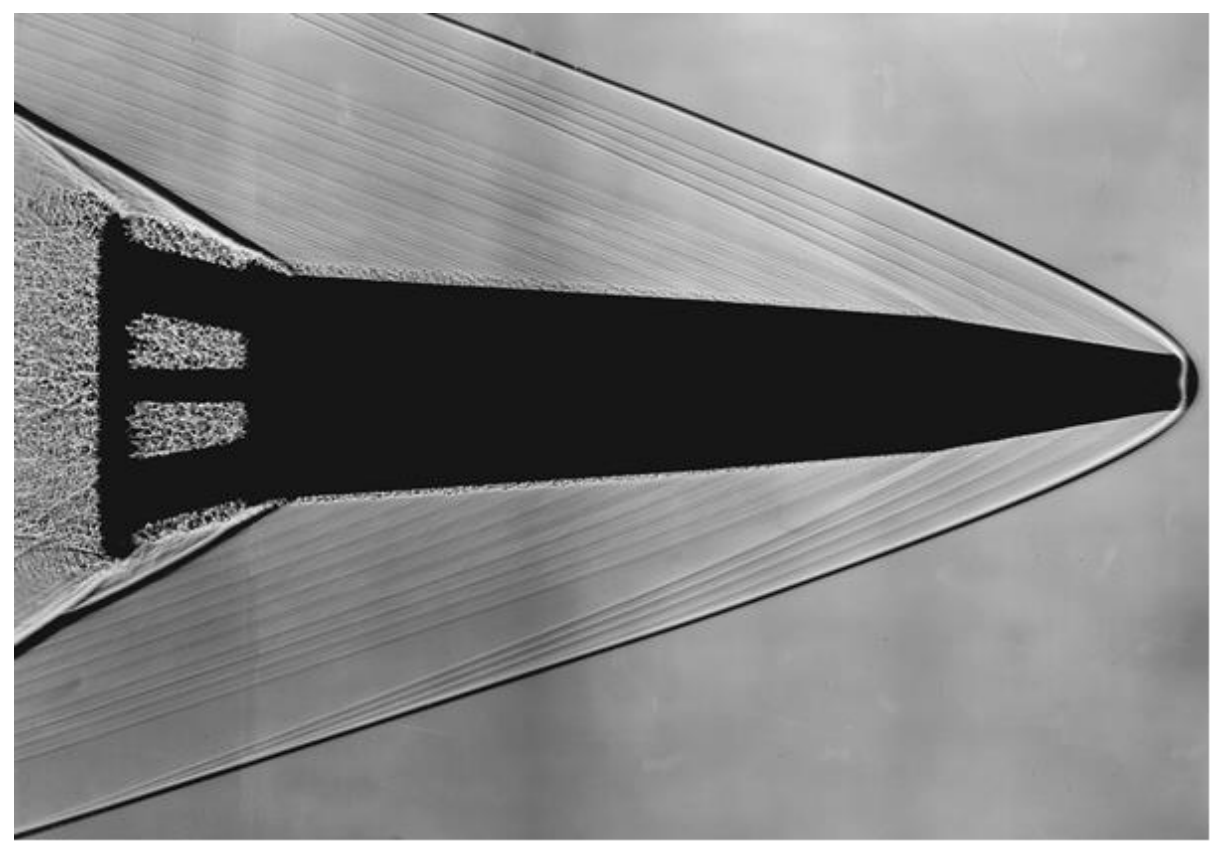

Figure 8 - Shadow spectrum of the flow around a model moving at supersonic speed.

The possibility of obtaining high-quality shadow images in the scheme of a luminous point in the conditions of operation of pulsed light sources that build images in reflected light on the sensitive elements of stereo post cameras (see above) is achieved by observing a number of conditions. First, a technical film with a maximum resolution (up to 500 pairs of lines per millimeter) is selected that is insensitive in the red region of the spectrum and has a low light sensitivity (several units). Secondly, the photographic material is covered with a film layer of rakord - a blue light filter that limits the possibility of exposure to the near ultraviolet-violet radiation spectrum [20]. Third, a powerful short point light source is used with a maximum of radiation in the ultraviolet part of the spectrum sufficient to expose a low-sensitivity film protected by a light filter with a blackening density higher than the veil [7]. Figure 9 shows the quality of the image obtained in one experiment in reflected light and the shadow picture of the movement of a system of bodies ( $V=1240 \mathrm{~m} / \mathrm{s})$.

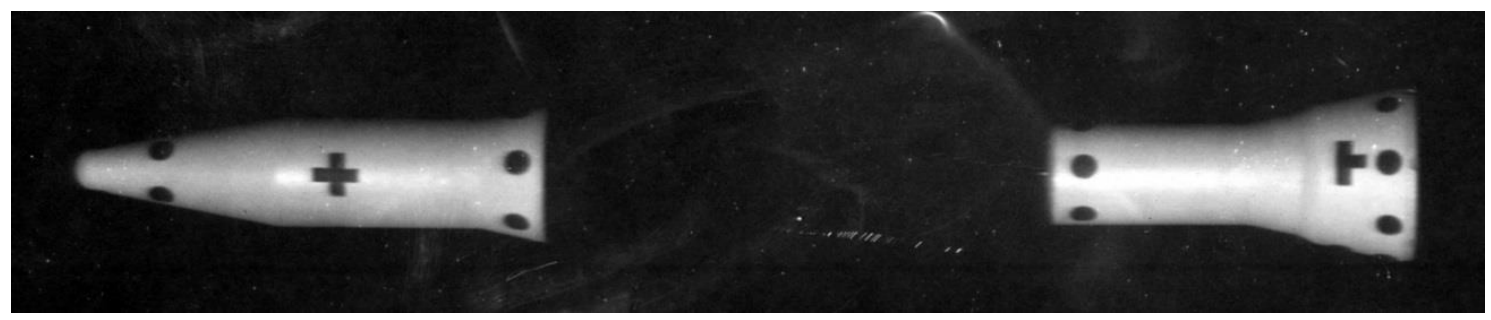




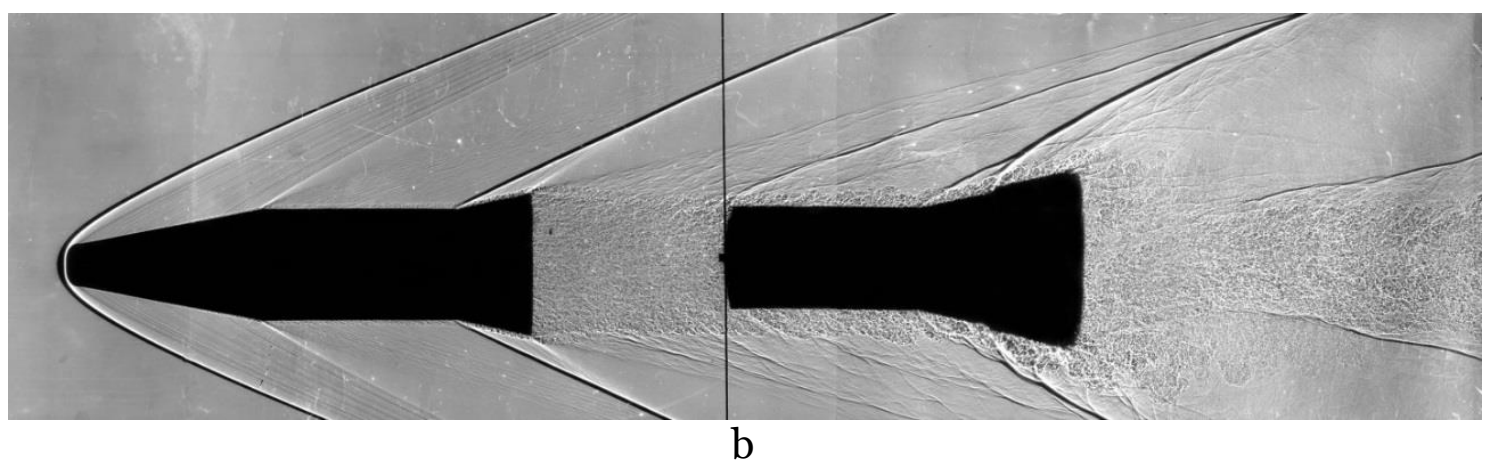

Figure 9 - Characteristic images obtained in one aeroballistic experiment for conducting the KP measurements(a) and analyzing the flow pattern (b) (vertical plumb line of the coordinate system).

Figure 10 shows the shadow spectrum of the flow around an asymmetric model with a negative pitch angle.

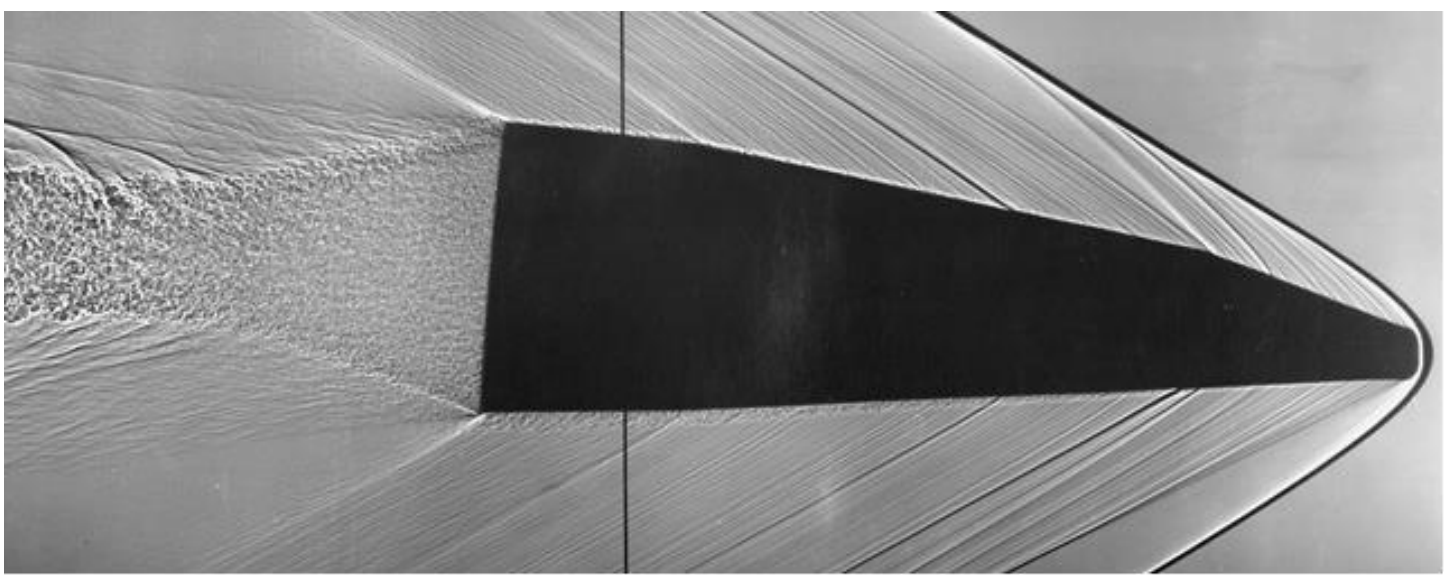

Figure 10 - Shadow spectrum of the model at $V \approx 790 \frac{\mathrm{m}}{\mathrm{s}}, \vartheta \approx-6^{\circ}, \gamma=-170^{\circ}$.

Figure 11 shows the shadow spectrum of a model with a panel control. The flow around the shield occurs with the formation of a zone of separation flow in front of it, an oblique shock wave (from the point of separation of the flow) and a more intense direct shock wave. The distance from the break-off point to the flap $\left(l^{\prime}=l / h_{u}\right)$ was 3.75 units with the slope of the oblique jump of the

seal $20^{\circ}$.

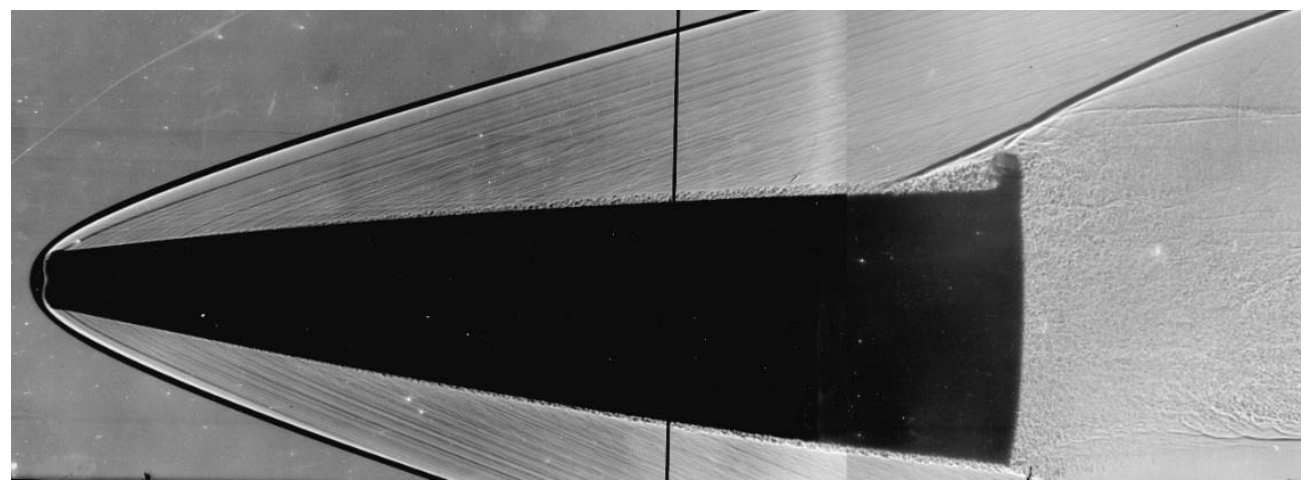

Figure 11 - Shadow flow spectrum of a model with a flap control, $V \approx 1410 \frac{m}{s}, \alpha \approx-1,5^{\circ}$,

$$
\gamma=-165^{\circ} \text {. }
$$




\section{Conclusion.}

The use of photoregistration in an aeroballistic experiment makes it possible to obtain high-quality shadow images of the flow spectra, which allow us to study the flow structures near the surface of the test model in flight. The advantage of the considered schemes is that they are used simultaneously with the solution of the basic problem-obtaining information (dozens of photo images of the marked model in reflected light) for external vector measurements, i.e. in conditions of "background illumination".

The work was carried out with the financial support of the RFBR (grants 18-o8-00715, 1829-10073)

\section{References}

1. Kasyanov V.A., Udartsev E.P. Determination of aircraft characteristics by identification methods. M. Mashinostroenie, 1988.

2. Zlatin N.A., Krasilshchikov A.P., Mishin G.I., Popov N.N. Ballistic installations and their application in experimental research. Nauka, GRFML, 1974.

3. Kastrov A.V., Shatilo A.M. Model-experimental methods for determining the aerodynamic characteristics of aircraft on ballistic routes. MO SSSR, 1982.

4. G.T. Chapman, D.B. Kirk. Method for Extracting Aerodynamic Coefficients from FreeFlight Data. AIAA Journal, No. 4, pp 753-758, 1970.

5. Zvegintsev V.I., Potapkin A.V. On development of aeroballistic range experiments for studying the acoustic shock// 2018, № 3, pp. 333-342

6. De Cecco, M., Francesconi, A. Impact facility based upon high frequency two-stage lightgas gun // Acta Astronautica, 2003, Volume 53, Issue 3, pp. 185-189

7. Gerasimov S.I., Faykov Yu.I., Kholin S.A. Cumulative light sources. Sarov, 2002.

8. De Cecco, M., Francesconi, A. Impact facility based upon high frequency two-stage lightgas gun // Acta Astronautica, 2003, Volume 53, Issue 3, p. 185-189

9. Gerasimov S.I., Lysenkov V.E. Launch and control system for optical and physical equipment on aeroballistic track. Collection of the 4th All-Russian Scientific and Technical conference "Fundamentals of ballistic design" St. Petersburg, 2014, p. 197.

10. Gerasimov S.I., Kostin V.I., Mende N.P. Algorithms for determining trajectory and aerodynamic parameters for moving revolution bodies: Handbook for University students SarFTI NRU MEPhI, 2013, $44 \mathrm{p}$

11. Patent 100313 of the Russian Federation, IPC Go3 B39/oo. Photopost combined for optical detection of fast processes. S.I. Gerasimov, K.V. Totyshev // Inventions. Utility model. 2010. № 34 .

12. Gerasimov S.I., Erofeev V.I., Salnikov A.V. Visualization of Muzzle Exhaust Following the Light Gas Gun Shot. Scientific visualization.Vol. 6. No 2, 2014.

13. Krutik M.I. Patent of the Russian Federation for utility model No. 63153, M. KL. Ho4N, 5/235, publish. 10.05.2007.

14. Raffel, M. Background-oriented schlieren (BOS) techniques. Exp Fluids 56, 60 (2015) https://doi.org/10.1007/s00348-015-1927-5

15. Bichal A., Thurow B. Development of a background oriented schlieren based wavefront sensor for aero-optics. Paper AIAA-2010-4842, 40th fluid dynamics conference and exhibit, Chicago.

16. Collection of articles. Edited by G. G. Cherny, S. Yu. Chernyavsky M. MSU publishing house, 1979.

17. N.P. Mende. On a method for determining nonlinear aerodynamic forces and moments. Collection of articles. Physical and gas-dynamic ballistic research. L., Nauka, 1980. PP. 200-224.

18. Shkvornikov P.N., Platonov N.M. Experimental ballistics. Devices and methods of ballistic measurements. Sofia: VVV VTS publishing House, 1976. 
19. Method for determining the speed of a throwing object: Pat. 2692116 ROS. Federation: SEC Go1P 3/38, Go1S 5/18 / Gerasimov S.I., Fomkin A.P., Trepalov N.A. No. 2018132081; application 06.09.18; publ. 21.06.19, bull. No. 18 .

20.Method for determining the speed of the object throwing a conical shape of large elongation: Pat. 2702955 RF: SEC Go1P 3/38, / Gerasimov S.I., Fomkin A.P., Trepalov N.A. No. 2018132358; claimed. 10.09.18; publ. 14.10.19, bull. no. 29.

21. System of shadow photographing of a fast-flowing process: patent no. 76166 of the Russian Federation/ Gerasimov S. I., Mende N. P., Latyshev K. V., pub. 10.09.2008 Byul. no. $25-5 \mathrm{~s}$ 